EPJ Web of Conferences 59, 13004 (2013)

DOI: $10.1051 /$ epjconf/20135913004

(C) Owned by the authors, published by EDP Sciences, 2013

\title{
X-ray calibration facility for plasma diagnostics of the MégaJoule laser
}

\author{
S. Hubert ${ }^{\mathrm{a}}$ and V. Prévot \\ Commissariat à l'Énergie Atomique, DAM, CESTA, BP. 2, 33114 Le Barp, France
}

\begin{abstract}
The Laser MégaJoule (LMJ) located at CEA-CESTA will be equipped with $\mathrm{x}$-ray plasma diagnostics using different kinds of $\mathrm{x}$-ray components such as filters, mirrors, crystals, detectors and cameras. To guarantee LMJ measurements, detectors such as x-ray cameras need to be regularly calibrated. An x-ray laboratory is devoted to this task and performs absolute x-ray calibrations for similar x-ray cameras running on Laser Integration Line (LIL). This paper presents the $\mathrm{x}$-ray calibration bench with its $\mathrm{x}$-ray tube based High Energy x-ray Source (HEXS) and some calibration results. By mean of an ingenious transposition system under vacuum absolute $\mathrm{x}$-ray calibration of $\mathrm{x}$-ray cameras, like streak and stripline ones, can be carried out. Coupled to a new collimation system with micrometric accuracy on aperture sensitivity quantum efficiency measurements can be achieved with reduced uncertainties.
\end{abstract}

\section{INTRODUCTION}

The LIL at CEA-CESTA near Bordeaux is a LMJ laser line prototype and therefore a laser-matter interaction plasma source facility as well. To analyze the corresponding x-ray emission spectrum different kinds of plasma diagnostics are used around the interaction chamber of this facility. They enable the study of plasma by several techniques such as x-ray imaging (time resolved or integrated), $\mathrm{x}$-ray spectrometry and by $\mathrm{x}$-ray transmitted or reflected energy measurements. To record the plasma $\mathrm{x}$-ray emission the LIL facility is equipped with detectors like $\mathrm{x}$-ray CCD, $\mathrm{x}$-ray streak and $\mathrm{x}$-ray framing cameras. For a correct interpretation of measurements performed with plasma diagnostics using these detectors it has been shown [1] that they need to be regularly calibrated before each experimental campaign. To achieve this goal we have been developing a new x-ray calibration laboratory since 2008 which aims to carry out absolute x-ray calibration on these kinds of cameras with the continuous concern of reducing uncertainties. All calibrations use therefore an x-ray tube based continuous source called High Energy x-ray Source (HEXS) with an anode wheel providing x-ray emission ranging from $0.9 \mathrm{keV}$ to $10 \mathrm{keV}$. This paper describes the HEXS calibration bench with some results obtained on $\mathrm{x}$-ray detectors and comparisons with theoretical models.

\section{HEX SOURCE AND CALIBRATION BENCH}

The x-ray source we use is a multi-anodes x-ray tube based continuous source developed by the "Optique and Électronique Appliquée" company consisting of an electron gun and an anode wheel set inside a vacuum chamber maintained permanently at a pressure of around $10^{-7} \mathrm{mbar}$ in operation. The watercooled anode wheel is hexagonal shaped each side being equipped with a different material ( $\mathrm{Al}, \mathrm{Ti}, \mathrm{Cr}$, $\mathrm{Cu}, \mathrm{Mo}, \mathrm{Ag}$ ) to obtain $\mathrm{x}$-ray characteristic lines ranging from 0.9 to $10 \mathrm{keV}$.

\footnotetext{
${ }^{\mathrm{a} e}$-mail: sebastien.hubert@cea.fr
}

This is an Open Access article distributed under the terms of the Creative Commons Attribution License 2.0, which permits unrestricted use, distribution, and reproduction in any medium, provided the original work is properly cited. 


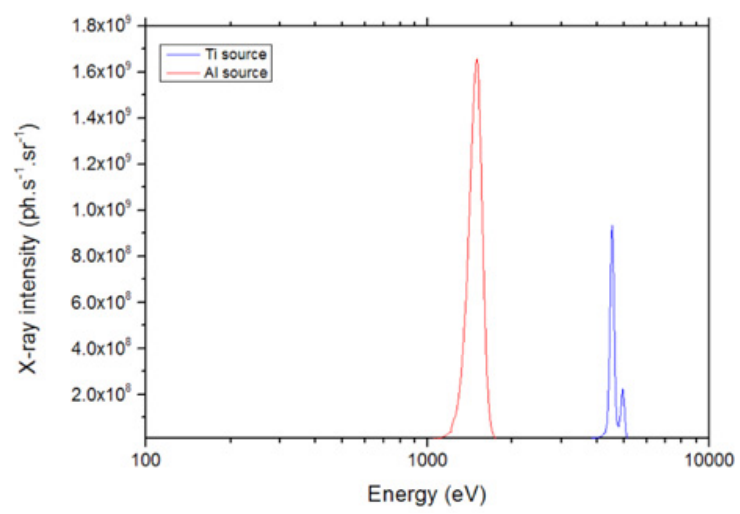

Figure 1. Spectra of $\mathrm{Ti}$ and $\mathrm{Al} \mathrm{x}$-ray source recorded by the Ge spectrometer.

The X-ray detectors calibration with HEXS is essentially performed using these radiation lines determined by the choice of anode and controlled by the current and voltage of the x-ray tube. The emission spectra are monitored by means of a Canberra GUL0035 Ultra LeGe germanium (Low energy germanium) liquid-nitrogen cooled spectrometer (Figure 1). This kind of spectrometer Ge acts as a photon counter and the charge collected by the Ge crystal is converted and processed by preamplifier/amplifier, analogic-digital coder and multichannel analyser stages. The energy calibration of the Ge spectrometer is achieved by means of x-ray intensity calibrated radioactive sources $\left({ }^{55} \mathrm{Fe}\right.$, ${ }^{65} \mathrm{Zn},{ }^{87} \mathrm{Sr}$ and ${ }^{109} \mathrm{Cd}$ ). The efficiency of the Ge spectrometer is now determined by calculation, including manufacturer specifications, and enables the X-ray intensity measurement of the HEXS sources in terms of photons per second and per steradian.

In front of the Ge spectrometer the x-ray beam is collimated (under vacuum) by a step by step motorized JJ X-ray collimation system using four blades to form vertical and horizontal slits. The typical used aperture is $88 \times 88 \mu \mathrm{m}^{2}$. To get the accurate knowledge of this aperture the motor step of this system has been measured to be equal to $1,04 \pm 0,06 \mu \mathrm{m}_{(\mathrm{k}=2)}$ (to be compared to $0.892 \mathrm{~mm}$ given by the manufacturer) by means of an $x$-ray imaging experiment using a great magnitude $(\gamma \approx 30)$. This great accuracy on motor step leads the relative uncertainty on the photon collection solid angle onto the spectrometer to reach $0,15 \%_{(\mathrm{k}=2)}$. Such decreasing over the collection solid angle relative uncertainty enables the $\mathrm{x}$-ray flux to be measured with a relative uncertainty ranging from 2 to $9 \%$ depending on the considered x-ray source.

The purpose of the calibration bench is to perform absolute calibration of x-ray cameras that cannot be placed entirely within the vacuum enclosure. Until 2010 [1] we used radiation from the rotating anode in two different directions. The $\mathrm{x}$-ray source intensity measurement (the reference) was made in one direction using the Ge spectrometer, and the x-ray camera calibration was situated in the opposite direction. Because of inaccuracy inherent in correlating the reference photon flux (measured by the $\mathrm{Ge}$ detector) with the signal recorded by the x-ray camera, we developed a transposition system which puts (under vacuum) successively the Ge spectrometer and the x-ray cameras to be calibrated in the same $\mathrm{x}$-ray beam (Figure 2).

The charge collection recorded by x-ray cameras is then directly correlated to the reference x-ray photon flux detected by the spectrometer. In these conditions only one $\mathrm{x}$-ray source must be considered with the same emission features for both Ge spectrometer and x-ray cameras. Furthermore maintaining the vacuum $\left(2-7.10^{-7}\right.$ mbar) inside the $\mathrm{x}$-ray cameras during the calibration process, which needs to transpose successively the spectrometer with the x-ray camera, enables the latter to be maintained at high voltage and guarantees the accuracy of the camera calibration. Except for x-ray CCD cameras all the others are based on an x-ray photon-electron converter combined to an electron to visible 


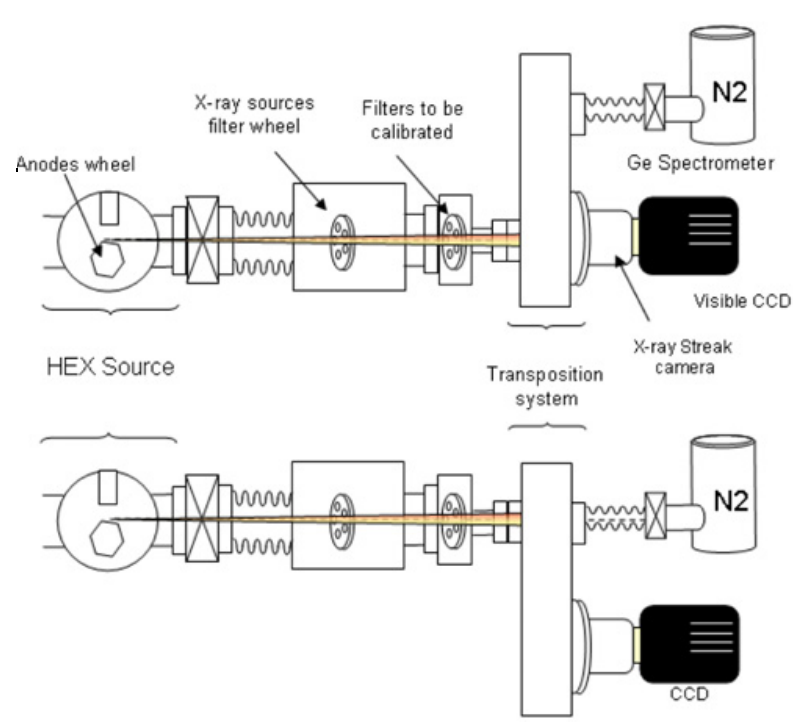

Figure 2. X-ray calibration bench principle with transposition system under vacuum.

photon phosphor screen. This visible emission is recorded by means of a quantum efficiency calibrated, backilluminated, $1300 \times 1340$ px, $20 \mu \mathrm{m}$ pixel size, Roper Scientific CCD.

\section{EXAMPLES OF X-RAY CALIBRATION WITH HEXS}

\section{1 x-ray streak cameras (XSC)}

The X-ray streak cameras used on LIL facility are based on a Photonis open tube with interchangeable CsI photocathodes to fit the experiment needs. These photocathodes allow XSC to cover the range $100 \mathrm{eV}$ to $10 \mathrm{keV}$. Calibrations performed on XSC consist in yield measurements over the HESX spectral range. We define this yield as the ratio of visible energy emitted by the phosphor screen to the incident HEXS X-ray energy and its measurement is carried out with XSC set in static mode for which electron beam is not deflected but focused at the center of the phosphor screen. Figure 2 shows results of X-ray calibrations of the LIL XSC\#5 equipped successively with hard (5000 $\mathrm{A}$ lexan/260 $\mathrm{Al} / 2400 \AA \mathrm{CsI})$ and soft $\mathrm{x}$-ray photocathodes $(1000 \AA$ lexan/260 $\mathrm{Al} / 2400 \AA \mathrm{CsI})$. Measurements are compared to a modified transmission Henke's model [2-4] and are in a relatively good agreement. Relative uncertainties achieved thanks to our transposition system and collimation system calibration range henceforth from 7 to $9 \%$ k=2 (squared plots on figure 3 ).

It is important to notice that random differences occur in photocathode behavior with regard to the model from one photocathode to another with a same XSC. Presented results show that yield differences can reach 5 to $27 \%$ over 1 to $5 \mathrm{keV}$ energy range which shows that absolute calibration of each XSC with photocathode must be carried out before experimental campaign.

\section{2 x-ray framing camera (XFC)}

These cameras consist of a gold alloy line-shaped photocathode deposited onto a microchannel plate (MCP) set in front of a phosphor screen. From the same manner HEXS performs yield absolute measurements of this kind of cameras. The same calibrated visible CCD is used to record visible emission coming from the phosphor screen. Figure 4 shows an example of yield calibration for these 


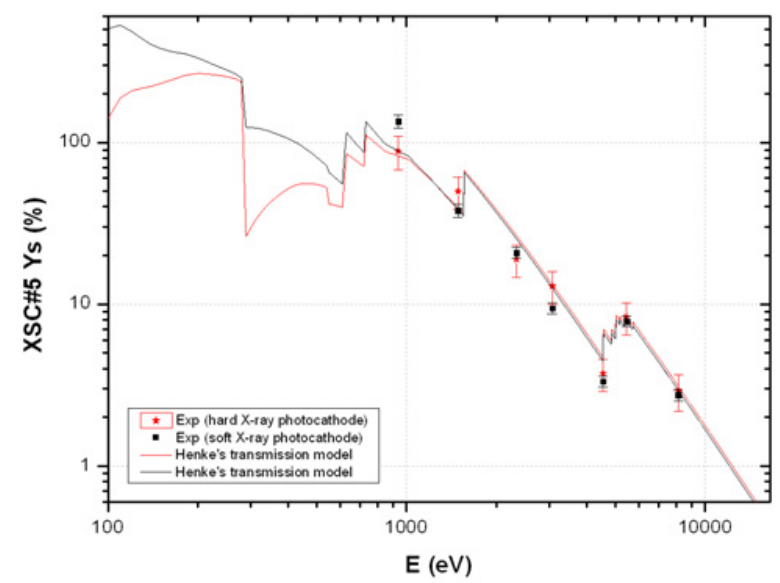

Figure 3. Yield measurements for one XSC with a hard and soft x-ray photocathode.

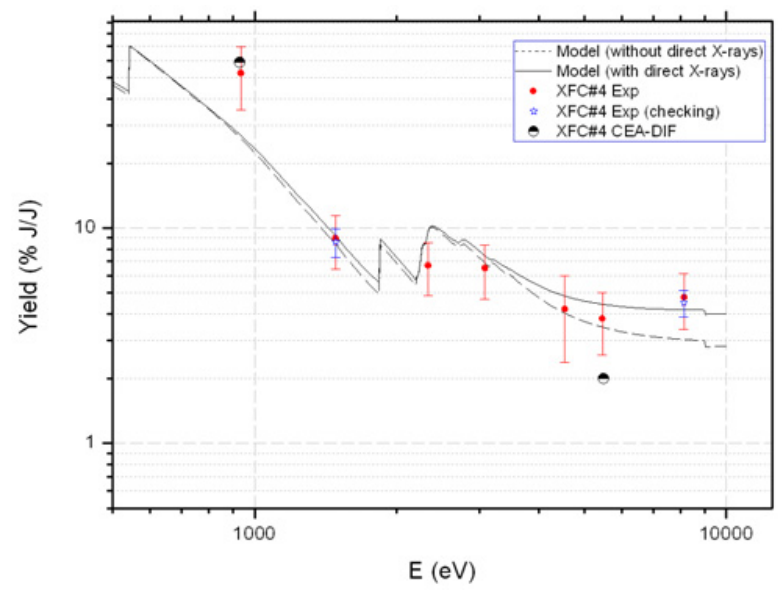

Figure 4. Yield measurements for XFC and comparisons between reference points (synchrotron correlated) and model.

cameras. Experimental data are compared to both CEA-DIF's work and one XFC model we developed which simulates the behaviour of MCP and phosphor screen in the calibration experimental conditions. This model takes into account the photocathode structure deposited on the MCP, the characteristics of the MCP (angle of the micro-channel with their diameter and length, MCP yield depending on the polarized voltage), as well as photoelectric yield of the photocathode and fluorescence yield of the phosphor screen.

On this curve the two CEA-DIF's points were performed with an x-ray tube and especially correlated to synchrotron's measurements [5]. A good agreement can be observed between our experimental results and these two reference points. We can see also that our model reproduces relatively well the XFC yield experimental behaviour. Further comparisons made with previous work show that our measurements reproduce qualitatively the XFC behaviour observed on LURE synchrotron [5]: in particular we observe the same rising of XFC yield at high energy. 


\section{IFSA 2011}

\section{CONCLUSION}

To guarantee the quality of measurements performed on LIL and LMJ facilities it is of great importance to regularly calibrate $\mathrm{x}$-ray cameras and components with weak uncertainties. The HEXS calibration bench is available to carry out these calibrations processes. It is used to calibrate the yield of X-ray streak cameras, x-ray framing cameras, quantum efficiency of x-ray CCD and filter transmission over the 0.9 to $10 \mathrm{keV}$ energy range. A particular effort has been recently made to reduce the measurement uncertainties by calibrating the collimation system in front of our reference spectrometer and by developing a system which transposes under vacuum successively this reference detector with the X-ray camera to be calibrated while maintaining it under HV supply. Henceforth yields of X-ray cameras are thus measured with an uncertainty ranging from 7 to $9 \%$.

\section{References}

[1] S. Hubert et al., Rev. Sci. Instr. 81 (2010)

[2] B. L. Henke, E. M. Gullikson, J. C. Davis, At. Data and Nucl. Data 52, 181 (1993)

[3] B. L. Henke, J. Liesegang, S. D. Smith, Phys. Rev. B 19, 3004 (1979)

[4] B. L. Henke, J. P. Knauer, K. J. Premaratne, Appl. Phys. 52, 1509 (1981)

[5] D. Gontier et al., SPIE 2869, 82 (1997) 